\title{
STUDY OF BASELINE CHARACTERISTICS AND 1-YEAR FOLLOW-UP OF DIABETIC AND NON-DIABETIC PATIENTS OF STEMI
}

\author{
Biswajit Das ${ }^{1}$, Trinath Kumar Mishra ${ }^{2}$
}

${ }^{1}$ Associate Professor, Department of Cardiology, SCB Medical College, Cuttack, Odisha.

2 Professor and HOD, Department of Cardiology, MKCG Medical College, Berhampur, Odisha.

\section{ABSTRACT}

\section{BACKGROUND}

Cardiovascular disease is currently responsible for $30 \%$ of all deaths worldwide with most of the burden now occurring in developing countries. ${ }^{1}$ DM is a risk factor for many cardiovascular diseases like Coronary Artery Diseases (CAD), Congestive Heart Failure (CHF) and atrial fibrillation.

Objectives- To analyse demographics, risk factors and treatment of diabetic vs. non-diabetic patients of STEMI admitted to a tertiary care hospital in India and subsequent 1-year follow-up of patients prescribed B blockers.

\section{MATERIALS AND METHODS}

This is a descriptive study of 284 consecutive patients of STEMI admitted to the hospital were included in the study. The patient's characteristics were recorded and the patients were followed up for 1 year.

\section{RESULTS}

Out of 284 patients, 232 (79\%) were non-diabetic and 62 (21\%) were diabetic. Out of all risk factors, hypertension was present more commonly in diabetics ( $55 \%$ vs. $33 \%, \mathrm{p}=0.03$ ). The 1 -year cardiovascular mortality was higher in diabetics on $\mathrm{B}$ blockers as compared to non-diabetics on B blockers (18.1 vs. 5.5\%, $\mathrm{p}=0.01$ ).

\section{CONCLUSION}

Diabetics with myocardial infarction are a high-risk patient population who are likely to have worse long-term outcome and require optimal therapy with diligent follow-up.

\section{KEYWORDS}

Diabetes, STEMI, Beta Blockers.

HOW TO CITE THIS ARTICLE: Das B, Mishra TK. Study of baseline characteristics and 1-year follow-up of diabetic and nondiabetic patients of STEMI. J. Evolution Med. Dent. Sci. 2018;7(04):515-518, DOI: 10.14260/jemds/2018/115

\section{BACKGROUND}

Cardiovascular disease is currently responsible for $30 \%$ of al deaths worldwide with most of the burden now occurring in developing countries. ${ }^{1}$ DM is a risk factor for many cardiovascular diseases like Coronary Artery Diseases (CAD), Congestive Heart Failure (CHF) and atrial fibrillation. The unabated rise of insulin resistance, obesity and metabolic syndrome is likely to increase the diabetic population 2-fold by $2030 .^{2}$ Diabetes mellitus confers an increased risk of death due to cardiovascular causes to the extent that two-third of adults with diabetes die of cardiovascular causes. ${ }^{3}$ Coronary Heart Disease (CHD) is much more common in diabetics than in the general population, affecting as many as 55 percent of these patients. The relative risk of Myocardial Infarction (MI) is $50 \%$ greater in diabetic men and $150 \%$ greater in diabetic women compared to age-matched non-diabetic subjects. Among patients admitted to coronary care units for AMI, $10 \%-20 \%$ are diabetic, the majority of whom have type 2 diabetes.

'Financial or Other Competing Interest': None.

Submission 13-11-2017, Peer Review 07-01-2018,

Acceptance 13-01-2018, Published 22-01-2018.

Corresponding Author:

Dr. Trinath Kumar Mishra,

Cardiologist,

Bhabha Nagar, $2^{\text {nd }}$ Line

Berhampur-760004,

Odisha.

E-mail:drtkmishra@yahoo.com

DOI: $10.14260 /$ jemds $/ 2018 / 115$

\section{(c) $($ ) $९$}

While the designation of CAD equivalent conferred on diabetes has remained debatable, patients with diabetes aggregate other comorbidities such as obesity, hypertension and dyslipidaemia which also contribute to increase the risk for CVD.

Beta blockers are an important component of the armamentarium of drugs prescribed to patients of myocardial infarction. A BARI 2D substudy with a follow-up period of upto 6 years concluded that in diabetic post MI patients, use of beta blocker is associated with reduced mortality. An observational study from France found that diabetics were actually prescribed $\beta$-blockers less often than non-diabetics with an absolute difference of $11 \% .4$ The present study was conducted to analyse the clinical characteristics of diabetic and non-diabetic patients hospitalised for STEMI and to compare the 1-year mortality of diabetic and non-diabetic patients on beta blockers.

\section{MATERIALS AND METHODS}

This is a descriptive study of 284 consecutive patients of STEMI admitted to the Cardiology Department, MKCG Medical College, Berhampur were analysed. The demographic details and risk factors of the patients were recorded. The treatment given during hospital stay and the drugs prescribed at discharge were documented. Those who were prescribed B blockers were then followed up for 1 year. Type 2 diabetes was diagnosed based on the need for treatment with insulin or oral hypoglycaemic drugs, a confirmed diagnosis of diabetes as per history or elevated fasting plasma glucose levels. Hypertension was diagnosed on the 
basis of history of hypertension, on treatment for hypertension or at least 2 measurements demonstrating elevated BP. Patients who had a smoking habit at present or consumed oral tobacco were considered smokers.

\section{Statistical Analysis}

SPSS software was used to perform all statistical analyses. The discrete and continuous variables were compared using Chi-square test and student ' $\mathrm{t}$ ' test. $\mathrm{P}$ value $<0.05$ was considered significant.

\section{RESULTS}

The characteristics of the patients are displayed in Table 1. Out of 294 patients, 232 (79\%) were non-diabetic and 62 (21\%) were diabetic. The mean age of the diabetic subgroup was 64.3 years, while that of the non-diabetic group was 66 years. $68 \%$ of diabetics were male as compared to $72 \%$ of non-diabetics. The incidence of smoking $(12.4 \%$ vs. $16 \%)$, hypercholesterolaemia (42.7 vs. 51.7\%) and sedentary lifestyle $(22.6 \%$ vs. $26.4 \%)$ was not significantly different between the 2 groups. Hypertension was significantly more common in diabetics than non-diabetics $(55 \%$ vs. $33 \%$, $\mathrm{p}=0.03)$. Past history of CAD was equally common in both groups (9.6\% vs. $12.6 \%)$. The frequency of thrombolysis was similar in both groups (28.6\% vs. $24.3 \%)$. An analysis of the drugs prescribed to the patients at discharge showed that anti-platelets, ACE inhibitors/ ARB and statins were prescribed to both groups at a similar high rate. Beta blockers were prescribed more frequently to non-diabetics as compared to diabetics, but the difference did not assume statistical significance.

44 diabetics and 198 non-diabetics were prescribed $B$ blockers. At the end of 1-year of follow-up, out of these patients, cardiovascular mortality was $18.1 \%$ (8 patients) in the diabetic group and 5.5\% (10 patients) in the non-diabetic group $(\mathrm{p}=0.01)$.

\begin{tabular}{|c|c|c|c|}
\hline Parameter & $\begin{array}{c}\text { Diabetics } \\
(62-21 \%)\end{array}$ & $\begin{array}{c}\text { Non- } \\
\text { Diabetics } \\
(232-79 \%)\end{array}$ & $P$ value \\
\hline Age & 64.3 & 66 & NS \\
\hline Male Gender & $4(68 \%)$ & $166(72 \%)$ & NS \\
\hline Hyper & & $76(33 \%)$ & 0.03 \\
\hline Smol & 8( & $38(16 \%)$ & NS (0.78) \\
\hline Known Case of & $6(9.6 \%)$ & $28(12.6 \%)$ & NS (1) \\
\hline Hypercholest & $26(42.7)$ & $120(51.7 \%)$ & NS $(0.41)$ \\
\hline Sedentary Lifestyle & $14(22.6)$ & $60(26.4 \%)$ & NS \\
\hline Thrombo & & $56(2$ & \\
\hline \multicolumn{4}{|l|}{ Drugs at Discharge } \\
\hline Antiplatelet & 621 & $226(97.9)$ & NS (1) \\
\hline ACEI/ ARB & $46(73.3)$ & $188(80.7 \%)$ & NS (0.45) \\
\hline Statin & $60(96)$ & $214(92 \%)$ & NS (0.69) \\
\hline \multicolumn{4}{|c|}{$\begin{array}{c}\text { Table 1. Baseline Characteristics and Treatment } \\
\text { Characteristics in Diabetic and Non-Diabetic Patients } \\
\text { of STEMI }\end{array}$} \\
\hline
\end{tabular}

\section{DISCUSSION}

Today, diabetes has become a worldwide epidemic. Myocardial infarction is a leading cause of mortality in diabetes. A major contributor to the deleterious effects of myocardial infarction is reactive oxygen species (ROS). 5 Hyperglycaemia by inducing mitochondrial dysfunction and endoplasmic reticulum stress increases ROS synthesis. ROS directly damages proteins, lipids and DNA and impedes intracellular signaling pathways. Hyperglycaemia also promotes endothelial dysfunction, pro-inflammatory and procoagulant factor expression. No release is defective, and apoptosis is augmented. Through such myriad and many other such unexplained pathways, diabetes influences the pathogenesis and outcome of CHD.

In our study, out of total 294 patients $79 \%$ were nondiabetics and $21 \%$ were diabetics. The incidence of DM in AMI varies from $10 \%-25 \%$. Our result is in agreement with previous Indian studies that have also demonstrated that diabetes is seen in $5 \%-20 \%$ of CAD patients. A recent study of 925 patients of ACS found $27 \%$ of patients to be diabetic, 6 whereas in the study by Sharma et al diabetics comprised $28 \%$ of ACS patients. ${ }^{7}$ The Kerala ACS registry found a higher incidence of $37.6 \%$ of diabetes. The International data reveals an estimate of $35 \%$ in the NCDR and $30 \%$ in the OASIS trial. 8 Hence, the importance of diabetes as a risk factor for CAD has been repeatedly underlined.

There was no gender difference between the two populations with both showing a male predominance. The incidence of hypertension was significantly more in the diabetic group $(\mathrm{p}=0.03)$. Vaur et al found hypertension in $70 \%$ of diabetics with MI as compared to non-diabetics. ${ }^{4} \mathrm{~A}$ German registry-based study also found an extremely significant increase in incidence of hypertension among diabetics. Also, among Indian studies, Sharma et al also found a significantly higher incidence of hypertension among diabetic patients of ACS as compared to non-diabetics (78.6\% vs. $44.4 \%, p=0.002) .7$ The combination of diabetes and hypertension significantly amplifies the risk of microvascular and macrovascular complications. Also, the relatively worse outcomes for patients of ACS from India as reported in the CREATE registry could be due to coexistence of multiple known risk factors. In contrast, Pandit et al found that hypertension was more prevalent in the non-diabetic category. ${ }^{9}$ Smoking was equally prevalent in both groups, which is in agreement with other Indian studies. ${ }^{7}$ A Korean study had similarly found no difference in incidence of smoking between the groups. ${ }^{10}$

The use of medicines for secondary prophylaxis was much more impressive compared to the large Indian registry data such as CREATE and Kerala ACS. An audit of physicians' prescriptions by Sharma et al in 2012 found that aspirin was used equally in both groups. ${ }^{11}$ However, ACE inhibitors/ARBs and statins were used more often in diabetics and B blocker use was significantly less. Our study found that all drugs were administered equally in both groups. B blocker use was lower in diabetics, but it did not reach statistical significance. This probably reflects the increasing adherence to guidelinedirected medical therapy. A contemporary Indian study has also found equal use of all drugs in both categories except dual antiplatelets and B blockers used less frequently in diabetics. ${ }^{7}$ Voller et al analysed registry data to show that statin therapy was administered equally in diabetics and nondiabetics (93\%) and similar was the case with aspirin $(80 \%))^{12}$

There is ample evidence to display that patients with DM have worse outcome than non-diabetics including both morbidity and mortality. The reasons include more extensive coronary artery disease, greater incidence of comorbidities and additional risk factors for CHD. Diabetics are also less 
frequently exposed to evidence-based therapy than nondiabetics. ${ }^{13}$ The mortality of diabetic patients on B blockers is significantly more than non-diabetics on B blockers. According to the landmark Framingham Heart Study, cardiovascular mortality is raised twice in diabetic men and 4 times in diabetic women as compared to non-diabetics. ${ }^{14}$ Diabetes not only plays an important role in causation of CAD, but also adversely impacts outcome following cardiac events. A subgroup analysis of diabetics with STEMI in the GUSTO 1 trial found higher 30 -day mortality in diabetics $(10.5 \%$ vs. $6.2 \%) .{ }^{15} \mathrm{~A}$ TIMI study group sub-study found that diabetes in ACS as compared to non-diabetics was associated with higher 1 year mortality in STEMI (13.2 vs. 8.1\%, p < 0.001) as well as NSTEMI $(7.2 \%$ vs. $3.1 \%, \mathrm{p}<0.001) .{ }^{16}$ Hur et al in a prospective Korean study of diabetic patients with MI found long-term mortality (after 1 month of stabilization) to be $3.9 \%$ in diabetics vs. $1.7 \%$ in non-diabetics $(\mathrm{p}<0.05) .17 \mathrm{~A}$ 2017 meta-analysis concluded that the risk of death overtime was significantly higher among diabetics compared with nondiabetics. ${ }^{18}$ This risk has been revealed in studies over the past 30 years and persists in spite of modern treatment strategies including reperfusion. Thus, in spite of all recent advances, the difference in mortality between diabetics and non-diabetics remains considerable.

In the present study, the use of B blockers was more common in non-diabetics, although not statistically significant. However, the 1-year cardiovascular mortality was higher in diabetics on B blockers. B blockers are known to have beneficial effects in post MI patients. In diabetics, an additional benefit of $\mathrm{B}$ blockers is the reduction of adverse events following severe hypoglycaemia. On the other hand, B blockers aggravate the potential risk of occurrence of severe hypoglycaemia and weight gain. A recent sub-study from the ACCORD trial found that in diabetics use of B blocker was associated with an increase in cardiovascular events, both in the larger category of all diabetics and in the smaller subgroup of patients with CHD or heart failure. ${ }^{19}$

Our study had a small sample size and was conducted at a single hospital. Hence, a larger study to confirm the findings and throw more light on the diabetic MI population would be in order.

\section{CONCLUSION}

Diabetics with AMI are considered high-risk patients. With the increasing prevalence of diabetes, this population of patients can only be expected to increase. A comprehensive understanding of the patient's profile and the grave prognosis of the disease will spur more intensive secondary prevention strategies to improve the long-term outcome.

\section{REFERENCES}

[1] Chiha M, Njeim M, Chedrawy EG. Diabetes and coronary heart disease: a risk factor for the global epidemic. Article ID 697240, International Journal of Hypertension 2012;2012: p. 7.

[2] Lipscombe LL, Hux JE. Trends in diabetes prevalence, incidence and mortality in Ontario, Canada 19952005: a population-based study. Lancet 2007;369(9563):750-6.
[3] Centers for Disease Control and Prevention. National Diabetes Statistics Report: estimates of diabetes and its burden in the United States, 2014. Atlanta, GA: US Department of Health and Human Services, 2014.

[4] Vaur L, Danchin N, Hanania G, et al. Management and short-term outcome of diabetic patients hospitalized for acute myocardial infarction: results of a nationwide French survey. Diab Metab 2003;29(3):241-9.

[5] Di Filippo C, Cuzzocrea S, Rossi F, et al. Oxidative stress as the leading cause of acute myocardial infarction in diabetics. Cardiovasc Drug Rev 2006;24(2):77-87.

[6] Shah BS, Deshpande SS. Assessment of demographics, treatment strategies and evidence-based medicine use among diabetic and non-diabetic patients with acute coronary syndrome: a cohort study. J Pharmacol Pharmacother 2014;5(2):139-44.

[7] Sharma KK, Mathur M, Lodha S, et al. Study of differences in presentation, risk factors and management in diabetic and nondiabetic patients with ACS. Indian J Endocr Metab 2016;20(3):354-8.

[8] Malmberg K, Yusuf S, Gerstein HC, et al. Impact of diabetes on long-term prognosis in patients with unstable angina and non-Q-wave myocardial infarction: results of the OASIS (Organization to Assess Strategies for Ischemic Syndromes) Registry. Circulation 2000;102(9):1014-9.

[9] Pandit A. Type 2 diabetes and myocardial infarction in central India: a study and review. Cardiology and Angiology: An International Journal 2017;6(1):1-14.

[10] Meisinger C, Heier M, von Scheidt W, et al. GenderSpecific short and long-term mortality in diabetic versus nondiabetic patients with incident acute myocardial infarction in the reperfusion era (the MONICA/KORA Myocardial Infarction Registry). Am J Cardiol 2010;106(12):1680-4.

[11] Sharma KK, Guptha S, Gupta R. Secondary prevention therapies for coronary heart disease in patients with type 2 diabetes: an audit. J Assoc Physicians India 2012;60:28-30.

[12] Völler H, Reibis R, Pittrow D, et al. Secondary prevention of diabetic patients with coronary artery disease in cardiac rehabilitation: risk factors, treatment and target level attainment. Current Medical Research and Opinion 2009;25(4):879-90.

[13] Norhammar A, Malmberg K, Rydén L, et al. Register of information and knowledge about Swedish heart intensive care admission (RIKS-HIA). Underutilisation of evidence-based treatment partially explains for the unfavourable prognosis in diabetic patients with acute myocardial infarction. Eur Heart J 2003;24(9):838-44.

[14] Garcia MJ, McNamara PM, Gordon T, et al. Morbidity and mortality in diabetics in the Framingham population. Sixteen year follow-up study. Diabetes 1974;23(2):105-11.

[15] Mak KH, Moliterno DJ, Granger CB, et al. Influence of diabetes mellitus on clinical outcome in the thrombolytic era of acute myocardial infarction: GUSTO-I Investigators (Global Utilization of Streptokinase and Tissue Plasminogen Activator for 
Occluded Coronary Arteries). J Am Coll Cardiol 1997;30(1):171-9.

[16] Cannon CP, McCabe CH, Gibson CM, et al. TNK-tissue plasminogen activator in acute myocardial infarction. Results of the Thrombolysis in Myocardial Infarction (TIMI) $10 \mathrm{~A}$ dose-ranging trial. Circulation 1997;95(2):351-6.

[17] Hur SH, Won KB, Kim IC, et al. Comparison of 2-year clinical outcomes between diabetic versus nondiabetic patients with acute myocardial infarction after 1month stabilization: analysis of the prospective registry of DIAMOND (DIabetic acute myocardial infarctiON Disease) in Korea: an observational registry study. Medicine (Baltimore) 2016;95(25):e3882.
[18] Gholap NN, Achana FA, Davies MJ, et al. Long-term mortality after acute myocardial infarction among individuals with and without diabetes: a systematic review and meta-analysis of studies in the postreperfusion era. Diabetes Obes Metab 2017;19(3):364-74.

[19] Tsujimoto T, Sugiyama T, Shapiro MF, et al. Risk of cardiovascular events in patients with diabetes mellitus on B-blockers. Hypertension 2017;70(1):10310. 